\title{
Experimental and genetic analysis of root development in Arabidopsis thaliana
}

\author{
Ben Scheres, Heather McKhann, Claudia van den Berg, Viola Willemsen, Harald Wolkenfelt, \\ Geert de Vrieze and Peter Weisbeek \\ Department of Molecular Cell Biology, Utrecht University, Padualaan 8, 3584 CH, The Netherlands *
}

Received 29 February 1996. Accepted in revised form 22 April, 1996

Key words: Arabidopsis, cell fate, embryogenesis, laser ablation, root meristem

\begin{abstract}
The cellular organisation of the Arabidopsis thaliana root is remarkably regular. A fate map of the primary root and root meristem that predicts the developmental destinies of cells within the embryonic root primordium has been constructed. Nevertheless, laser ablation experiments demonstrate that root meristem cells develop according to position and not according to lineage. Mutational analysis has identified genes required for cell specification in the radial as well as in the apical-basal dimension. The corresponding gene functions appear to be necessary during embryogenesis for the formation of a correctly patterned primary root.
\end{abstract}

\section{Introduction}

Roots have been studied in earlier decades by researchers interested in plant development, who noticed the ordered patterns of cell division and the resulting regularity of cell files. Early workers focused on understanding division patterns within root meristems. Histological studies provided evidence for the existence of a few "initial cells" from which all root tissues arose (e.g. Von Guttenberg, 1955). Thymidine labelling demonstrated the existence of a "quiescent centre" in root apices. Surgical experiments indicated the presence of multiple initials, and substantiated the concept of the "promeristem" in root apices: a quiescent centre surrounded by initials for all cell types (Clowes, 1961).

A question that could not be addressed at that time was whether the initial cells were specifically programmed for the formation of only one or few related tissues. The idea that regularly arranged meristem cells were programmed to form specific tissues was designated the "histogen" concept (Hanstein, 1870). A variety of cell division patterns in roots of different species have been described. Some division patterns provid-

* FAX No: +31302513655 ed clear evidence for separate initial cells for particular cell types. In other cases a single founder cell appeared to give rise to progeny of all cell types (cf. Steeves and Sussex, 1989). Yet other root apices displayed irregular cell divisions, in line with clonal analyses on shoot development that emphasised flexibility and the role of cell position rather then lineage in plant development (reviewed by Poethig, 1987). The underlying mechanisms of flexible shoot meristem development are yet to be elucidated. Whether cell specification in some, or all, root meristems operated via similar flexible mechanisms could not be answered.

At present, the methodology developed by animal developmental biologists has an increasing impact on developmental studies in plants (e.g. Jürgens, 1995; Weigel and Meyerowitz, 1994). This approach can take a new advantage of the regularity of root development to probe mechanisms of cell programming within meristems. In this chapter we present experimental and genetic analyses, carried out in the model plant Arabidopsis thaliana. The Arabidopsis root is very regular and excellently suited to exploit fully the available experimental and genetic tools. We have combined clonal analysis with cell ablation studies to study the flexibility of meristem initial cells, and the source of their developmental information. Furthermore, we 
present an analysis of mutations affecting the embryonic formation of the root meristem. These mutations point to a pivotal role of daughters from one embyonic cell, the hypophyseal cell, in establishing the primary root meristem. Taken together, these studies suggest how root meristem cells learn their fate and provide a genetic entry into how the meristem itself is organised during ontogeny.

\section{The Arabidopsis root promeristem has a fixed clonal origin}

The Arabidopsis primary root meristem contains a surprisingly constant number of cell files of each cell type that terminate in the initial cells. A small set of initials for all tissues surround four quiescent cells (Dolan et al., 1993). This quiescent centre contacts all the initials, an observation that suggests regulatory functions. Quiescent centre and initials together are termed the promeristem, the minimal construction centre of the root (Clowes, 1953). All cells within the promeristem are laid down during embryogenesis, and exhibit the division pattern typical for the root meristem from the heart stage of embryogenesis onward.

Two sets of initials within the promeristem can give rise to more than one differentiated cell type. The epidermal initial gives rise to both epidermis and lateral root cap, while the cortical initial forms endodermis and a cortical cell layer. These relations have been demonstrated by anatomical as well as sector analysis (Dolan et al., 1993, 1994). The rigidity of these relations superficially suggests that both types of initial cells and programmed by lineage to give rise to two alternative cell types.

We have performed clonal analysis to study how the primary root meristem is laid down during embryo development. We analysed embryonic sectors which arose by transposon excision from the uidA (GUS) marker gene in transgenic plants. The end points and width of these sectors allowed us to deduce a complete fate map for the Arabidopsis root (Figure 1A; Scheres et al., 1994). The root promeristem arises from two distinct groups of cells that are separated at the first zygotic division: the quiescent centre and columella root cap arise from the hypophyseal cell that is, in turn, derived from the basal cell, while the proximal initials arise from the apical cell (Figure 2). Apparently, the daughters of the hypophyseal cell come to cooperate with the proximal initials to give rise to the functionally integrated root meristem. The separation
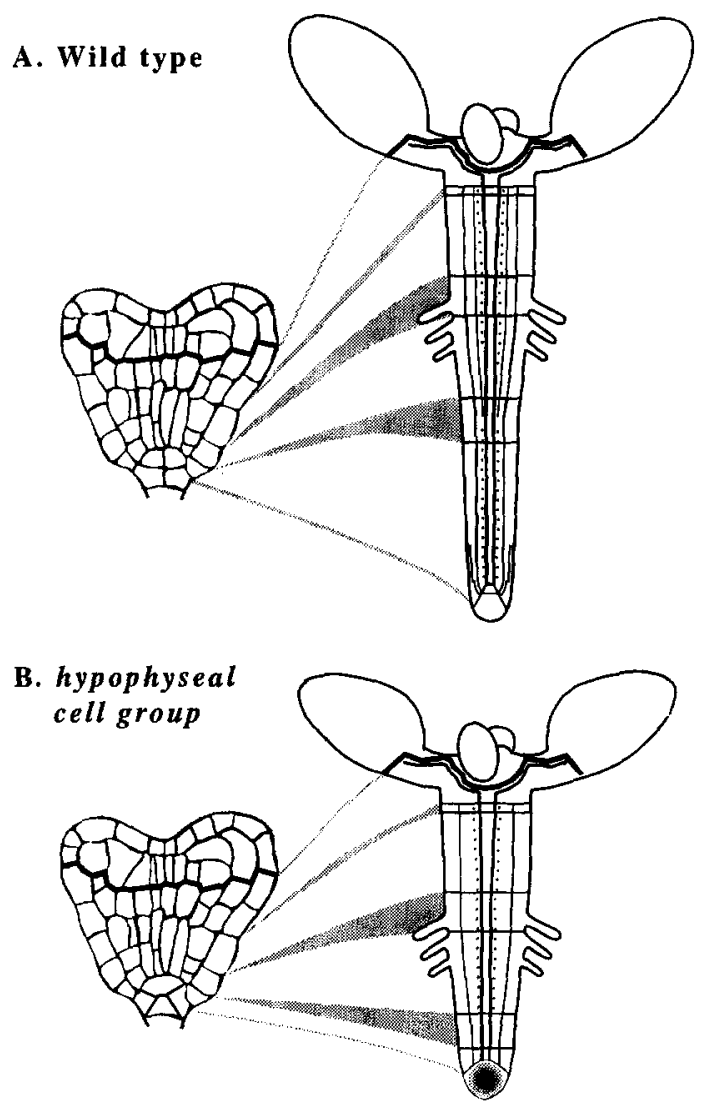

Figure 1. Fate map of wild type and hypophyseal cell group early heart stage embryo. Corresponding regions in embryo and seedling are connected by lines. Broadening of the connecting lines visualises the variability in the location of embryonic cell division planes in the seedling axis. Components of the seedling axis below the cotyledons from top to bottom: cotyledon shoulder, hypocotyl (ending basally just above the uppermost root hairs), embryonic root and meristematic root. (A) Wildtype embryo and seedling with all tissue types. (B) "Hypophyseal cell" mutant embryo and seedling lack cells with the anatomical features of quiescent centre and columella, and display no or limited proximal meristem activity.

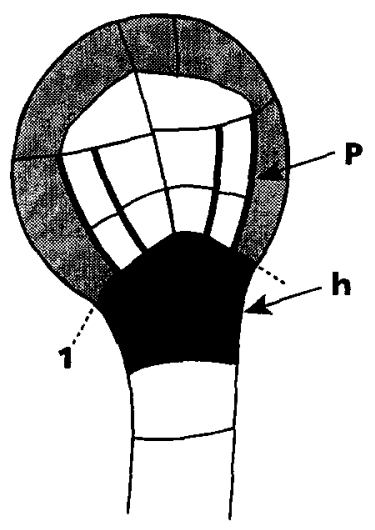

Figure 2. 16-cell embryo. P_protoderm, h_hypophyseal cell, I-plane of first zygotic division. 
of the main tissue types: protoderm, ground tissue and vascular cambium, also occurs early during embryogenesis (cf. protoderm in Figure 2). These divisions, like the first zygotic division, act as clonal boundaries that separate different cell fates. Root meristem initials are set apart from these tissues at a later stage. These observations are consistent with the hypothesis that the early divisions restrict cell fates to cell lineages; they are, however, in striking contrast to clonal analyses in the shoot meristem, where it was shown that, in general, no fixed relations exist between cell lineages and cell types.

\section{Probing the flexibility of initial cells by experimental manipulation: laser ablation studies}

The absolute correlation between cell type and embryonic lineage in the Arabidopsis promeristem could be explained in terms of lineage-dependent development. This would be in line with the classical concept of histogens as founder cells for specific cell types (Hanstein, 1870). On the other hand, positional signalling might determine cell fate. In this case, positional information would have to act at a single-cell resolution, since many layers in the root meristem comprise only one cell. Being superimposed on a rigid cell lineage, such a position-dependency would go undetected. To investigate which one of these two means for cell fate specification occurs in the Arabidopsis root, we performed laser ablation experiments.

In such experiments, one meristem cell is killed and a neighbouring cell can occupy its position. If the neighbouring cell ends up in a different tissue it crosses a clonal boundary. If commitment of cells were irreversible, the cells would develop into their "old" tissue type. On the other hand, if a continuous system of positional information were operative in the Arabidopsis root meristem, the fate of incoming cells after laser ablation would change.

Application of the fluorescent dye propidium iodine to roots of intact seedlings was followed by confocal laserscan microscope (CLSM) analysis. Propidium iodine is taken up within 10 minutes by living roots. The dye surrounds living cells without entering them, thereby outlining individual cells in optical sections of roots (Figure $3 \mathrm{~A}$ ). We ablated one or more individual cells by focusing the laser beam on a cell in a specific plane of focus. The addition of propidium iodine presensitised the cells; the effect of the laser pulses on unstained roots was more then an order of magnitude less. The fluorescent compound entered successfully ablated cells and resulted in cytoplasmic staining (Figure 3B). The induced permeability was not transient. If propidium iodine was again added after growing the roots for a prolonged time without the dye, the ablated cells could be re-stained (Figure $3 \mathrm{C}$ ). Without new addition of dye no CLSM image was obtained, showing that cytoplasmic staining of ablated cells was due to the entrance of newly added propidium iodine. After prolonged growth of the root, ablated cells became completely compressed (Figure $3 \mathrm{C}$ ). In the majority of cases, Remnants of ablated cells retained a fluorescent signal after fixation, embedding and sectioning.

We investigated the ability of root meristem cells to switch fate when they cross the boundary set by the first zygotic division (i.e. the separation between the quiescent centre and columella on the one hand, and the proximal meristem on the other hand). Upon ablation of quiescent centre cells, the underlying (more distal) columella cells ceased to divide. As a result of this, the dead quiescent centre cells were carried off distally and cell files, continuous with the vascular bundle, were displaced toward the root tip. By using promoter marker gene fusions specific for vascular bundle and columella, we have shown that these displaced cells switch fate, and display columella-specific gene expression instead of the former vascular-specific expression (Van den Berg et al., 1995). Therefore, the clonal boundary set by the first zygotic division does not restrict the developmental potential of the resulting daughter cells.

Upon ablation of cortical and epidermal initial cells, the dead cells are compressed toward the periphery of the root and cells from more internally located tisues take up the position of the ablated cell. We recorded the fate of such cells that are forced to develop in an adjacent tissue layer. Pericycle cells invading the cortical cell layer were capable of switching fate and formed both endodermis and cortex. Cortical initials invading the epidermal cell layer formed both epidermis and lateral root cap (Van den Berg et al., 1995). We concluded that proximal root meristem cells, despite being clonally restricted to tissue layers at early stages of embryogenesis, are flexible in fate. Hence the early embryonic divisions in the radial plane, like the first zygotic division in the apical-basal plane, are not instrumental in restricting developmental potential. Our experiments are in line with indirect evidence that has been obtained by clonal and chimera analysis of the shoot meristem (e.g. Poethig, 1987), and demonstrate that positional 

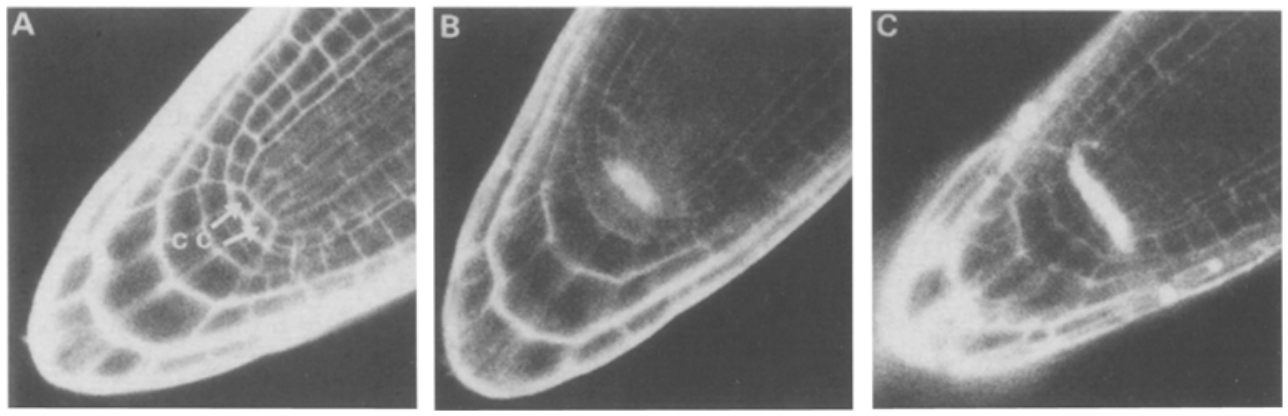

Figure 3. Roots of seedlings stained with propidium iodine $\left(10 \mu \mathrm{g} \mathrm{mL}^{-1}\right.$ for $10 \mathrm{~min}$. at room temp. and visualised by CLSM (mrc-600, Bio-rad; Zeiss Axiovert). cc_central (quiescent centre) cells. (A) Root of seedling 2 days after germination. (B) Same root. 10 min. after ablation of the quiescent centre. Propidium iodine has entered the quiescent centre cells. (C), seedling root 3 days after ablation of the quiescent centre.

information is acting in the regularly patterned root meristem as it is in the shoot.

\section{The source of positional information for the radial pattern of root tissues}

The position-dependent development of root meristem cells immediately puts forth the question of the source of the positional information. We have investigated the dircetion of positional cues that guide the differentiation of cortical initial cells in both endodermis and cortex. The typical division pattern of the initial is to divide transversally to give rise to a proximal daughter. This proximal daughter divides asymmetrically to produce cortex and endodermis. How is the cortical initial instructed to perform this division? Laser ablation of the one proximal and its two neighbouring cortical daughters that are connected with one initial cell, isolates one cortical initial. We have shown that such an initial no longer performs the typical asymmetric division (Van den Berg et al., 1995). On the other hand, ablation of three proximal cells connected with the initial in another plane ( 1 epidermis, 1 cortex, 1 pericycle) did not interfere with the asymmetric division. We concluded that positional cues, specifying cortical and endodermal cell fate, are perpetuated distally in the cortical/endodermal cell layer. This observation could be extrapolated to the other tissue layers in which we have shown that flexibility in cell fate exists. In that case, a model becomes conceivable in which more mature regions of the root serve as a signalling source and hence as a patterning template for the differentiation of meristem cells.

Which genes are involved in generating the signals that allocate cells to tissues in the root? A number of mutants have been described that alter the radial organisation of the root (Benfey et al., 1993; Scheres et al., 1995). Every mutant in this class defines a different genetic locus. Given the allele frequencies of the identified loci and the difficulty with which the phenotype of radial pattern mutants can be predicted, probably most of these genes are as yet unidentified. The three mutants shortroot, scarecrow and pinocchio are affected in the specification of cortex and endodermis from the ground tissue. wooden leg and gollum interfere with the specification of the vascular tissue. Noteworthy, the layer-specific phenotypes persist in the hypocotyl, and all five mutants have an embryonic phenotype throughout the embryonic axis. Hence pattern information is delivered distally to the meristem initials, and all genes identified so far that influence the pattern affect the complete seedling axis from embryogenesis onward. The tentative conclusion is that the information in the root meristem originates as the result of gene activities during embryonic radial pattern formation. Thus, the radial pattern of cell types emanating from the root meristem most likely reflects the activity of multiple embryonic genes that specify many different elements of the radial pattern.

Cells in the distal region of the root, containing the quiescent centre and the root cap, are programmed differently since they do not form elements of the radial tissue pattern specific to the seedling axis. Yet the data on signalling in the apical-basal axis show that this region is programmed by positional information. In the next section we will present genetic data that suggest the great importance of this distal region in establishing a root meristem. 


\section{Mutational analysis of root meristem specification}

In addition to radial specification of cell layers, the root and root meristem are specified as elements of the apical-basal embryonic pattern. The clonal analyses showed that the boundary between the root and the hypocotyl does correlate, but not with cellular precision, to early embryonic divisions (Fig. 1A; Scheres et al., 1994). This indicates that, during early pattern formation, root and hypocotyl fate are connected. A few Arabidopsis genes have been described which are required for the formation of a root. Among these, the MONOPTEROS gene (Berleth et al., 1993) has been analysed in detail, as discussed elsewhere in this volume. This gene appears to be required for the specification of both root and hypocotyl in the embryonic context. The $m p$ phenotype also indicates the intimate relation between root and hypocotyl specification.

We have concentrated on defining loci which are involved in the programming of a correctly patterned root meristem. The fate map of the Arabidopsis seedling shows that the majority of the primary root cells arise from the root meristem. However, cells within a small region covered with root hairs that connects root and hypocotyl originate from a different region in the embryo and are referred to as the "embryonic root". We performed a genetic screen for mutant seedlings that contained the embryonic root, but lacked an organised promeristem. Upon EMS seed mutagenesis, 18,000 independent $\mathrm{M} 1$ families were screened for the segregation of seedlings with defective root meristems. Siblings of putative mutants were checked in the next generation for re-segregation of the phenotype, grouped according to seedling phenotype, and analysed by complementation analysis. In this chapter we will discuss in some detail four genetic loci that upon mutation confer embryonic defects in the hypophyseal cell region (e.g. the prospective quiescent centre and columella): HOBBIT, BOMBADIL, ORC, and GREMLIN (Table 1; Figures 4, 5). A number of less well studied mutants were identified, which have similar phenotypes to the ones stated above but are not allelic (Table 1). All the mutants have in common that they are fully recessive and seedling-or adult lethal.

\section{The hobbit phenotype group}

The HOBBIT locus is defined by a series of independent allelic mutations which lead to a very similar, "root meristemless" appearance (Figure 4). Sev- en independent alleles were identified in our screen, and four more alleles were kindly provided by Prof $G$ Jürgens (Univ Tübiningen, Germany) and Dr H Höfte (INRA Versailles, France). Seedlings homozygous for strong $h b t$ alleles display no root meristem activity, while seedlings homozygous for weak alleles allow some residual activity. All seedlings homozygous for $h b t$ alleles have abnormal root meristem anatomy. The most conspicuous anatomical defects are the irregularities in cell shape, number, and arrangement of the columella and quiescent centre region. Mutants homozygous for strong $h b t$ alleles contain no differentiated columella root cap, based on the absence of starch granules. $h b t$ mutants carrying either strong or weak alleles show abnormalities in the division pattern of the hypophyseal cell from early globular stage embryo onward. These abberations seem restricted to the hypophyseal cell at early stages of embryogenesis, but the proximal initials, most notably the epidermal initials that should form a lateral root cap, can also become abnormal (Figure 5A). This mutant phenotype suggests a function of the $H B T$ gene in 1) specifying hypophyseal cell progenitors; 2 ) the triggering of activity in the proximal meristem, and 3 ) the proper formation of a lateral root cap.

The existence of one complementing mutant with features strikingly similar to the $h b t$ mutants opens up the possibility that more genes, acting very close in the hierarchy of hypophyseal cell specification, exist. Careful phenotypic analysis and molecular epistasis studies, once cloned genes become available, will aid in the establishment of the relationship between these genes with perhaps identical phenotypes.

An important feature of the $h b t$ mutants is that the root phenotype is not embryo-specific. Adventitious roots from $h b t$ mutants, generated from the hypocotyl of seedlings or via tissue culture, have the characteristic mutant phenotype and arrest development. Therefore the $H B T$ gene, unlike the $M P$ gene, is not required just for embryonic root formation but for root formation in all developmental contexts (Berleth et al., 1993).

\section{The bombadil phenotype group}

Further evidence for more than one gene acting in a similar specification pathway comes from the embryo phenotype of the $b b l$ mutants. $b b l$ seedlings are distinguishable from $h b t$ seedlings (Figure 4 ), and they form a different complementation group with different map position than hbt. While the seedling phenotypes are 
Table 1. Hypophyseal cell group genes. All loci (a minimum of seven and maximum of sixteen) are grouped in the first column. Abbreviations: the number of independently recovered alleles per locus, and linkage to one of the five Arabidopsis chromosomes are listed in subsequent columns

\begin{tabular}{llll}
\hline Locus & Abbreviation & Alleles/Locus & Chromosome \\
\hline HOBBIT & $H B T$ & 11 & 2 \\
BBL-likel & & 1 & N.D. \\
BOMBADIL & BBL & 2 & 3 \\
BBL-like 1,2,3,4,5 & & N.D. & N.D. \\
ORC & ORC & 1 & 5 \\
GREMLIN & $G R M$ & 1 & 5 \\
ORC-like 1,2,3,4,5,6 & & N.D. & N.D. \\
\hline
\end{tabular}

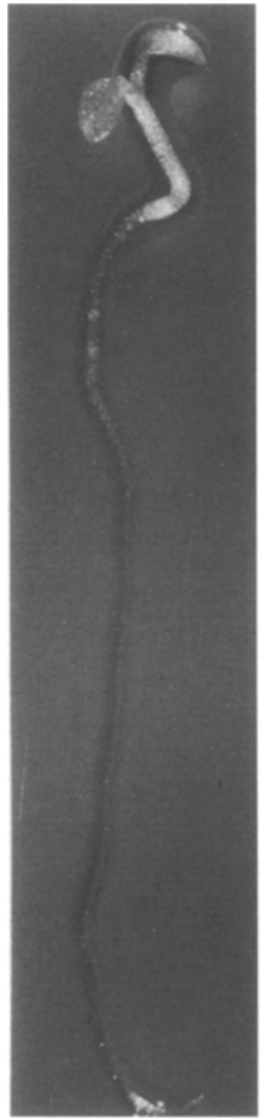

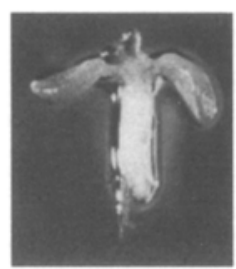
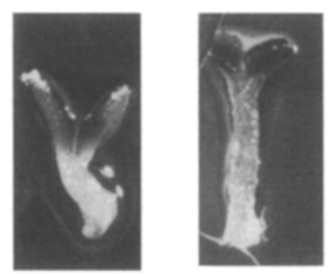

hobbit

$b b l$

orc

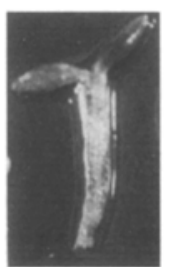

gremlin

Figure 4. Seedling phenotype of wildtype and "hypophyseal cell group" mutants; All mutants are photographed at the same magnification, except wildtype $(\approx 80 \%)$.

distinct, the anatomical deviations in globular and heart stage $b b l$ mutant embryos are similar to those observed in $h b t$ mutant embryos. At the mature embryo stage, the shape of $b b l$ root poles differs. The typical epidemal initial division leading to a lateral root cap layer appears to be present in $b b l$ mature embryos (Figure
5), as well as in the secdling. It has yet to be confirmed whether these presumptive root cap cells, and the cells in the position of the wildtype columella, acquire all root cap characteristics. As with the hbt phenotype class, a number of non-allelic mutations exist which give rise to seedlings closely resembling $b b l$ seedlings 


\section{MATURE EMBRYO STAGE}

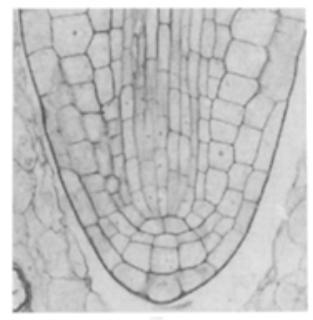

WT

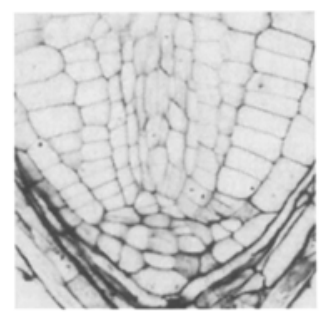

$h b t$

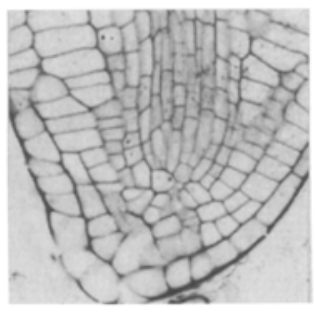

$b b l$

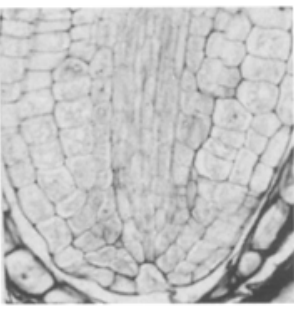

orc

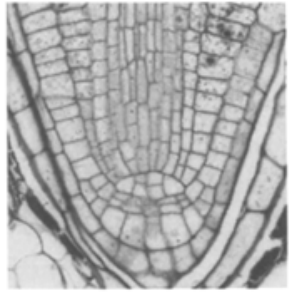

grm

Figure 5. Mature embryo phenotype of wildtype and "hypophyseal cell group" mutants. Astra-Blue stained sections of Technovit-embedded seeds.

(Table 1). These will need to be subjected to mapping and pairwise complementation analysis, and the embryonic phenotype will have to be fully described. From such studies the meaningfulness of the "seedling phenotype group" classification should become apparent. At present, we have identified at least a number of genes involved in closely related aspects of hypophyseal cell specification. The amount of saturation cannot yet be assessed.

\section{The orc phenotype group}

The mutants of the orc phenotype group share a similar seedling phenotype, of which the reduced root is the most conspicuous (Figure 4). For none of these mutants is an allelic series available yet, which hampers somewhat the analysis of the phenotype. Under the assumption that the mutants represent full loss-of-function, the $O R C$ and GREMLIN genes, which interfere with the formation of the hypophyseal cell descendants at least from the heart stage onward, are not solely responsible for the acquisition of meristematic potential: limited meristem activity is observed. The embryonic phenotype is always conspicuous in the hypophyseal cell region, but includes variable anatomical features. In orc embryos and seedlings, sometimes the cell in place of the wildtype hypophyseal cell appears not to have divided at all (Figure 5). In other cases, randomised divisions in the orc hypophyseal cell region result in a cluster of small cells at the position of the hypophyseal cell descendants. The latter anatomical features are also characteristic for gremlin seedlings.

The observed variability in division patterns interferes with simple classification of the orc group of mutants. The use of promoter/enhancer trap lines will be essential to understand fully the nature of the defect in these mutants. Mutants specific to hypophyseal cell programming would be expected to have altered tissuespecific marker gene expression in this but not other regions of the embryo/seedling. Alternatively, some of the orc group mutants may be explained by a higher sensitivity of the hypophyseal cell to mutations in the machinery for cell division. The extreme regularity of hypophyseal cell divisions in comparison with the embryo proper may put more stringent requirements on the division apparatus within these cells during embryogenesis.

\section{Concluding remarks}

A combination of clonal analysis and laser ablation studies has revealed a striking flexibility in cellular fate within the rigidly patterned Arabidopsis root meristem. Apparently, the precision and predictability of the tissue pattcrn is not the consequence of regular cell divisions establishing determined cell lineages, but of the precision of a system of positional cues on top of a regular cell division pattern. Analysis of mutations in the FASS gene, in which division patterns are deregulated from early embryogenesis onward confirm this idea. Despite the abnormalities in division, the pattern elements in the root are essentially present (Torres Ruiz and Jürgens, 1994). The organismal perspective of plant development states that cell division patterns are not primarily instrumental in morphogenesis (e.g. Kaplan and Hagemann, 1991). Our observations extend this view to organs with precisely deter- 
mined patterns of cell division like the Arabidopsis root. This notion makes one feel more at ease when contemplating the beforementioned diversity in cell division patterns in root apices. These differences may reflect for example mechanica/physical differences in cell wall parameters, which lead to different but highly stereotyped patterns of division (cf. Hejnowicz and Hejnowicz, 1991). If the mature region of the root acts as a template to specify the cell files that emerge from widely different meristems, it is no longer necessary to imply differences in cell specification mechanisms in different root types, regardless of the organisation of the meristem initial cells. Moreover, flexibility in cell fate within both root and shoot apices may point to common mechanisms by which the cells in the two meristems are programmed.

The hypophyseal cell descendants in the distal region of the root do not respond to the radial pattern information that is present in the root meristem. How important is this distal end for root meristem programming? The genetic analysis of root meristem specification suggests a primary role of the hypophyseal cell descendants in the formation of the root meristem, since all root meristem formation mutations studied in detail show embryonic defects in this region, and appear to lack at least some of the cell types formed by the hypophyseal cell in the seedling. At present, it may be inferred that the $h b t$ and $b b l$ phenotype group genes are required at a somewhat earlier stage in embryogenesis then the orc phenotype group genes. Phenotype analysis solely based on anatomy may be misleading however. Gaining some understanding as to how these genes act in the specification of the root meristem will require analysis of marker gene expression in the different mutant backgrounds, double mutant analyses, and molecular analysis of at least a number of the genes that have now been identified. As exemplified by the $h b t$ allelic series, an incorrectly specified hypophyseal cell appears not only to affect the formation of cell types (e.g. root cap) but also the potential for post-embryonic divisions that is characteristic for the root meristem. Recently, the $R M L 1$ and $R M L 2$ loci were described (Cheng et al., 1995). Mutations in these genes appear not to affect embryogenesis but result in the absence of cell divisions specifically in the root meristem. It will be interesting to assess the molecular relationship of these genes to the ones that are the subject of this chapter.

We have shown that the root cap and quiescent centre region of the Arabidopsis root are specified continuously by a system of positional information. Further- more we have identified genes in the "hypophyseal cell group" that are essential for the specification of these cell types. It remains to be seen whether molecular information on the hypophyseal cell group genes will establish a picture of cell wall components that guide cellular differentiation and pattern formation (Berger et al., 1994), of soluble signals that pass through plasmadesmata which are themselves organised in a specific way (cf. Duckett et al., 1994), or of yet another mechanism of signalling to provide a functionally integrated and flexible root meristem.

\section{References}

Van den Berg C, Willemsen V, Hage W, Weisbeek P and Scheres B 1955 Cell fate in the Arabidopsis root meristem determined by directional signalling. Nature 378, 62-65.

Berger F, Taylor A and Brownlee C 1994 Cell fate determination by the cell wall in early Fucus development. Science 263, 14211423.

Berleth T and Jürgens G 1993 The role of the monopteros gene in organising the basal body region of the Arabidopsis embryo. Development $118,575-587$.

Cheng J-C, Seeley K A and Sung Z R 1995 RMLl and RML2, Arabidopsis genes required for cell proliferation at the root tip. Plant Physiol. 107, 365-376.

Clowes F A L 1961 Apical Meristems. Blackwell, Oxford, UK.

Dolan L, Janmaat K, Willemsen V, Linstead P, Poethig S, Roberts $\mathrm{K}$ and Scheres B 1993 Cellular organisation of the Arabidopsis root. Development 119, 71-84.

Dolan L, Duckett CM, Grierson C, Linstead P, Schneider K, Lawson E, Dean C, Poethig S and Roberts K 1994 Clonal relationships and cell patterning in the root epidermis of Arabidopsis. Development $120,2465-2474$

Duckett C M, Oparka K J, Prio D A M, Dolan L and Roberts K 1994 Dye-coupling in the root epidermis of Arabidopsis is progressively reduced during development. Development $120,3247-3255$.

Guttenberg H v, Burmeister J and Brosell H-J 1955 Studien uber die Entwicklung des Wurzelvegetationspunktes der Dikotyledonen II. Planta 46, 179-222.

Hanstein J 1870 Die Entwicklung des Keimes der Monocotyledon und der Dikotylen. Bot. Abhandl. (Bonn) 1-112.

Hejnowicz and Hejnowicz K 1991 Modeling the formation of root apices. Planta 184, I-7.

Jürgens G 1995 Axis formation in plant embryogenesis: cues and clues. Cell 81, 467-470.

Kaplan D R and Hagemann W 1991 The relationship of cell and organism in vascular plants. Bioscience 41, 693-703.

Poethig R S 1987 Clonal analysis of cell lineage patterns in plant development. Am. J. Bot. 74, 581-594.

Scheres B, Wolkenfelt $\mathrm{H}$, Willemsen V, Terlouw M, Lawson E, Dean and Weisbeek P 1994 Embryonic origin of the Arabidopsis primary root and root meristem initials. Development 120, 24752487.

Scheres B, Di Laurenzio L, Willemsen V, Hauser M-T, Janmaat K, Weisbeek P and Benfey P N 1995 Mutations affecting the radial organisation of the Arabidopsis root display specific defects throughout the embryonic axis. Development 121, 53-62. 
Steeves T A and Sussex I M 1989 Patterns in plant development. Cambridge Unjversity Press, Cambridge, UK.

Torres-Ruiz R A and Jurgens G 1994 Mutations in the FASS gene uncouple pattern formation and morphogenesis in Arabidopsis development. Development 120, 2967-2978.
Weigel D and Meyerowitz E M 1994 The ABCs of floral homeotic genes. Cell 78, 203-209.

Section editor: $H$ Lambers 